\title{
EL LÉXICO DE LAS VIÑAS EN JEREZ DE LA FRONTERA*
}

ABAJADOR: "sombrero de palma que se coloca encima del mortero que contiene el arranque, una vez se le eche el agua caliente).

ABIERTA DE LA CEPA : "parte del tronco de la cepa donde comienza el nacimiento de los brazos».

AEOTONAR: "brotar la yema o botón en primavera».

ABRIR : "manifestar la vid sus flores».

ABRIR UN CUADRANTE: "cavar un hoyo para reponer la planta perdida o seca. Se hace cuando la viña está ya poblada y se ha perdido una cepa).

ACODAR: "preparar un sarmiento para poder proceder con él, en su día, a la plantación en acodon.

AgARRAR : "echar raíces la cepa).

Agostado: ((acción de agostar).

AGOSTAR: "primera operación de limpieza que hay que hacer al plantar una viña. Se lleva a cabo en el mes de agosto y tiene por finalidad limpiar la tierra de malas hierbas y simientes, rompiendo y desmenuzando el terreno con azuela o con arado de malacaten.

AgUaderas : “al mazón de palos que sirve para transportar cántaros en las caballerías).

* En Homenajes. Estudios de Filología Española, Madrid, 1964 (I) y 1965 (II), tengo publicadas las dos partes complementarias de este trabajo, Tonelería (I, páginas 43-55) y Bodegas (II, págs. 69-89). En mi Contribución léxica a la geografía dialectal, Madrid, 1965, doy una extensa bibliografía sobre esta materia. Quiero, desde aquí, agradecer al erudito conocedor de tantos temas jerezanos, don José de Soto Molina, la amabilidad y saber con que siempre ha atendido y disipado dudas y vacilaciones. 
AguA NIEBLA : "lluvia más fina que la agïita caladora y más gruesa que la niebla).

AgÜITA CALAdORA : "lluvia finísima».

AHORQUILLAR : "afianzar o asegurar las varas de las cepas con horquillas para que no se desgajen con el peso de los racimos».

Ahoyar: "abrir hoyos o baúles para la plantación de vides».

AJO CALIENTE: "comida hecha a base de pan asentado, cortado o pellizcado a mano, que se condimenta con aceite, ajo, sal, y al servirse se le agrega agua caliente y pimientos cortados a tiras) $\| \mathrm{Cf}$. Cuestionario ALEA, 674.

Alamaduras : "tierra de buhedo en que aparecen restos de antiguas construcciones).

Alambrado : "cerca de alambres").

Albarizas : "terrenos calcáreos, compactos y gredosos, que se distinguen por su color claro o albo. Mal llamadas las afueras —pues no todas las afueras son albarizas-; es terreno de primera calidad».

Albero: Véase Albarizas.

Albillo : "especie o variedad de uva blanca".

AIINEAR: "formar líneas las vides al plantarlas".

Almijar : "explanada descubierta, con orientación al mediodía, que suelen tener las casas de las viñas y en donde se solea la uva antes de pisarla).

Alomar : “levantar cavando lomos de tierra para formar los cuadros de las vides)".

Arlanar : "poner llana o igual la superficie de la viña. Se hace una vez se haya esponjado el terreno con las lluvias de otoño e invierno que deshacen los grandes terrones de la superficie).

AMARRAR PALOS : "atar a un tutor o rodrigón los brazos de la cepa para evitar que los vientos la desgajen. Esta expresión se usa más que la de amarrar los pulgares y menos que la de amarrar verdes).

AMARRAR los PUlgares: Véase AMARRAR PALOS.

AMarrar Verdes : Véase Amarrar palos.

AMo: "nombre que dan los braceros de la viña y obreros de la bodega al dueño de la propiedad" |! Cf. Cuestionario AI.EA, 530.

AMORRONAR : "arropar con tierra el injerto de yema".

ANGARILlAS : “especie de jaula de madera, más ancha por arriba que por abajo, que sirve para transportar las uvas en la vendimia).

APALEAR: «mezclar y remover los racimos de uva dentro del lagar para mejor efectuar la pisa).

ApISONAR : "triturar o machacar los racimos con pisones". 
Aplomada: "dícese de la uva cuyo color tira a verde grisáceo». APORCADO: "abrigado de las cepas).

Aporcadura : "montón de tierra con que se abrigan las cepas y que procede de las piletas o lomos deshechos después de las primeras lluvias". APORCAR : "amontonar, en invierno, determinada cantidad de tierra alrededor del tronco de la vid para resguardarla de las heladas".

ARADO Malacate: "(tipo de arado con el cual se hacía la agosta".

ARANZiDA: "medida de superficie usada para las viñas).

ARENAS : "tierras de color amarillento, más o menos rojizas por el óxido de hierro, de formación suelta o arenosa, si bien no exactamente lo que se entiende por arena suelta».

Argulema : "nudo donde nace la yema".

ARRANCAR : "quitar la cepa).

ARRANCAR DE PATILLA : "quitar la cepa de raíz".

Arranque: Véase Ajo CALIENTe.

ARRENDAR: ((alquilar).

ARRIERO: "hombre que lleva las angarillas».

ARROPAR LA INJERCIÓN: "cubrir de tierra el injerto".

ASEAR LA CEPA: "limpiar la cepa arrancando con la mano y radera tuda la cáscara vieja, seca o podrida, huevecillos de insectos, etc.l.

ASENTAR: "allanar la superficie de la viña».

AsErira: "pileta cuadrada, con cuatro lomos de tierra, formada alrededor de la cepa para retener las aguas de lluvia"

ASERPIAR : "abrir aserpias".

AZADA: (instrumento con el que se abren los cajones, de lámina ancha y cabo recio y muy inclinado hasta formar ángulos de $50^{\circ}$ con la cuchilla).

AzOLETón : "pequeña azada que llevan los capataces de viña para corregir cualquier defecto en las labores de los obreros»).

AzUFRADO : "acción de ahumar con azufre las vides para prevenir muchas enfermedades".

BAJEAR EL, VIENTO: “estar flojo el viento queriendo entrar y acabar con el existente).

BAJO: "terreno fangoso".

BARBADO : "sarmiento ya enraizado -es decir, con raíces-, dispuesto para la plantación").

BARBAR : (enraizar el sarmiento».

BARBAS: "raíces que nacen en la cepa del país, impidiendo que engorde el patrón). 
BARBón: "cepa nueva) || "sarmiento plantado en el vivero para que eche raíces" || "sarmiento con raíces que nace casi de la raíz de la planta".

BARRA : "palanca o eje de hierro, terminado en cruz por su parte superior y en punta por la inferior, que se usa para plantar los sarmientos una vez hecho el marcadon.

BARros : "tierras de color pardo, más o menos oscuras, compuestas de de. pósitos de descomposición orgánica; dan abundantes mostos, aunque no limpios y finos".

BASTARDEAR : "degenerar, echarse a perder las castas o variedades de las vides cultivadas").

BAÚlES: "hoyos o cuadrantes de un metro cúbico donde se echa estiércol. Se abren en el centro de cada cuatro cepas).

BEBA : "variedad de uva, gorda pero no uniforme en su tamaño, ovoidea, basta, pero jugosa y algo ácida».

BIENTEVEO : "construcción provisional hecha en la viña, generalmente en la parte más elevada o estratégica, desde donde el viñadero ahuyenta a los que quieren robar uvas. Está formada por una empalizada o cañizada cubierta de hojarasca seca y puesta sobre altos palos para darle elevación y dominar el horizonten.

BINA : "cuarta labor que se hace a las viñas; se corta y asienta la tierra con la azada varias veces hasta que queda compactan.

BINAR : "comprimir, apretar, asentar la tierra para que no quede ahuecada).

BLANQUEAR : "pintar las cepas con cal o yeso blanco para prevenir enfermedades" $\|$ Cf. Cuestionario AlEA, 590.

Boca : "hoja larga, estrecha y delgada de la hoz de podar con la que se cortan los sarmientos».

Botón : "hinchazón que denota la futura yema» \| "yema».

BRAzoș : "sarmientos gruesos que nacen de la cabeza»).

BUEN CERNIDO: "abundante fecundación".

BUEN DESPOJO : "abundante fecundación".

BUHEDO: "tierra áspera, negra, muy fértil, compuesta de una mezcla de albarizas, barros y arenas; ocupa las cañadas y faldas de las colinas de albariza; los calores del verano producen en ellas enormes hendiduras)!.

BURRERo : "obrero último de la fila".

CABERNET: "clase de vidueño, muy rubio".

Cabeza : "parte del tronco de la cepa donde comienza el nacimiento de los brazos».

CAÍDA DE LA HOJA: (deshoje natural de la vid». 
CAJONES: "hoyos que se cavan en la viña para en ellos plantar la cepa". Cajuela : "taladro hecho en el patrón donde encajará la yema, en el injerto de yema).

CALITA: "trozo suelto del racimo con unas cuantas uvas".

CAMADA: "anchura o separación que media entre las cepas de una viña, es decir, el espacio entre liño y liño".

Canasta : "cesto redondo y ancho de boca, hecho de mimbre, caña o vareta de olivo, empleado en la recolección de la uva. Aproximadamente tiene capacidad para una arroba de uval.

CANASTo: "cesto redondo, de boca ancha, de mimbre o caña, usado para recoger el rebusco, llevándose al brazo" || (tcolador que se pone en la piquera para impedir que el mosto arrastre el hollejon.

Canuto: "parte del sarmiento entre dos nudos o yemas".

Cañocazo: "casta de uva" || vidueño de hojas peludas y amarillentas, fruto muy dulce, redondo y dorado, hoy día casi eliminado su cultivo por su acentuada predisposición a estar corrido".

CAPACHA : "canasto hecho con pleita".

Capacho: "disco tejido de esparto, sin asas, con vuelta y orificio en el centro, en el que se vacían las tınetas y canastas de uva para que, expuestas éstas al sol, se soleen en los almijares de la viña; su diámetro es de casi un metro y puede contener una arroba de racimos que rinden aproximadamente 7 litros de moston.

Capataz : "obrero jefe de los que trabajan en las faenas de la viña, a cuya vigilancia quedan encomendadas todas las labores del cultivo de la vid».

CAPATAZ DE CORBilio : "obrero que eventualmente, en la viña, durante la vendimia, vigila la corta de la uva y organiza el acarreo de las canastas al almijar, porque el capataz está en el lagar dirigiendo las faenas de la pisan.

CAPIRote: "trozo mal labrado de las viñas).

Carajo a ia veia : Véase Juan de la Viña.

Carajote: Véase JuAN dE LA Viña.

CARRETADA : "medida de capacidad equivalente a unas sesenta arrobas".

CASA LAGARES: "local en el edificio de la viña en el que existe un lagat y donde se efectúa el pisado y prensado de la uva».

CÁSCARA: "corteza superflua que hay que quitar para evitar que sea el cobijo de parásitos y hongos».

CASERO: "persona al frente de la casa de la viña; nada tiene que ver con el capataz: es cocinero, limpia, etc.".

Castlitas : "baúles, hoyos». 
Castelilano: "variedad de uva".

CASTELIANO GARRIDO: "variedad de uva".

CASTEÑUla : "planta perjudicial que daña considerablemente las viñas porque sus raíces taladran las raíces de las cepas" "| Cyperus Longus L.

CAstiza: Véase Esquirmeña.

CASTRA : "acto de castrar».

CASTRAR: "quitar a la cepa los tallos, yemas y sarmientos tiernos que no son fructíferos o que han salido en la garganta de la planta o en mal sitio de su cabeza; así se deja a la cepa libre de chupones y se favorece el vigor de los buenos sarmientosm "| Cf. Cuestionario AleA, 1106.

Catana : "pan, aceite y vinagre» \|V Véase Vinagrilıo.

Cavabien: "labor consistente en romper o deshacer las piletas y lomos abiertos en la labor de aserpiarn.

Cava litana: Véase Golpe Lleino.

CAVADOR: "obrero que cava las viñas".

CAVAR: "trabajar la tierra).

Cencerrilito: Véase Rebuscos.

Cencerrones : Véase ReBuscos.

Cennizo: "nombre que se le da al Oidium (Uncinula Necator); produce una mancha más o menos oscura en el sarmiento y un polvillo en la uva que lo agrieta y pudren.

CEPA : "planta leñosa, sarmentosa, de cuyo fruto - la uva- se obtiene el vino" \| (tronco de la vid». Nunca se dice en Jerez vid, sino cepa. VITIS VITIVINIFERA L.

CEPA ABIERTA : "cepa seca).

Cépa CARRAsqueña: "cepa endeble).

Cepa con coraje: "vid frondosa).

Cepa esquilmeña: "vid que produce mucho fruto".

CEPA DE PALOMINO BASTO: "vid que nunca llega a madurar».

CEPA VIRGEN : "planta sarmentosa parecida a la vid».

CEPORRO : "cepa grande".

CERnIDo: (efecto de cerner la vid, es decir, de caer la flor y. quedar el fruto» $\| \mathrm{C} f$. Cuestionario AlEA, 1108.

Cernidurá: "cisco menudo que se obtiene de los sarmientos de las vides cuando se podan las viñas; es de fácil combustión, no produce humo $y$, por ello, se usa para el brasero".

Cerrrillo : "planta perjudicial, parecida al lastón".

Cigarrón: (iinsecto dañino que come los tallos de la planta, nunca las uvası.

Cisco : Véase Cerrnidura. 
Crorosis : "enfermedad de la cepa que se traduce por la coloración amarilla de sus hojasn.

Cochina: Véase Marranı.

Cogouro: "hojas de palma usadas para liar el injerto".

Corodrmio: "cabeza de la cepa".

Cororo: "labrador de alguna heredad de viña por arrendamiento; vive en ella).

COrOR DE ORO: "tonalidad que indica sazón o madurez en la uvà.

Corlzón : "parte carnosa o jugosa de la uva".

CORIZÓN DE CABRITO: "variedad de nua de forma entrelarga".

CORAZÓN DF, IA IFẼ : "parte interna del sarmiento o de la cepa por donde circula la savian.

CORRENTINES: "gavias naturales hechas por las aguas de lluvia".

CORRIDO: Véase ĖsTAR CORRIDO.

CORTA DE IA UVA: "operación realizada desde finaies de agosto hasta mediados de septicmbre, consictente en separar los racimos de la cepa segín su grado de madurez»).

COR'TAR: "hendir la cepa al podarla; estos cortes hay que pintarlos con sulfato de hierro" !" "arrancar la cepa de! viñedo".

CORTIR ios muMONes: "arrancar los hijuelos o retoños que nacen de las raíces de las cepas).

CORTAR I.OS VERDES: "cercenat los sarmientos".

Costero: "obrero de la viña, destacado por sus compañeros para ir periódicamente a la cindad llevando y trayendo ropa, tabaco, comestibles, etc.).

Costo: "nan y menestra -garbanzos, aceite, vinagre...- que diariamente el costero lleva a la viña deste la cindad".

Cruz: Véase CABeza.

Cruzados: "lomos formados nor la tierra que se extrae al hacer la pileta y que constituyen los cuatro lados de éstan.

CUADRA: "lugar para los mulos".

CUADRANTE: "hoyo en el que se planta un sarmiento".

CUARTEIAR: "distribuir e! terreno en partes, en cada una de las cuales se plantan distintos vidueños para observar cuál da más fruton.

CUBETA: "cuba perneña usada en la viña para sacar agua del pozo».

Cunig.1: "acto de colgar la uva para consumirla mucho después de haber sido recolectadan.

Culirio: "cerco más o menos marcado que suele rodear el punto de inserción de la uva en el nezón" "! "pequñõo agujero que resulia en la uva por la separación del pezón'm. 
Chapó: "sarmiento que crió la uva el año pasado y descansa este año".

Chasserlas : "casta de vidueño más oscuro que el cabernet).

Chata : "acción de abrir hoyos o piletas en torno a las cepas».

Chatear : "labor antigua que consiste en hacer los lomos entre dos obreros, de forma tal que uno hace - de los cuatro lomos de la piletados contiguos, y el otro obrero los dos restantes".

Chaveilla : Véase Zagal.

Chicharra : "insecto dañino que come la uva verde pero no en agraz». Chiquillo: Véase Zagal.

DAR DE MANo: "dejar de trabajar, descansar».

Depósitos De mosto: Véase Pocejón.

DERBARBAR : "cortas las barbas".

Desbragadura : "hoyo o cava profunda hecha en los pies de la cepa para que recoja el agua de lluvia y conserve la humedad".

DESBRAGAR : "desbaratar la tierra amontonada alrededor del tronco de la cepa» || (dejar al aire el patrón injertado para cortarle los mamones" \| "cortar las barbas".

DescabezAR : "cortar los sarmientos a la altura conveniente para que sólo broten las yemas necesarias para la formación futura de la cepan.

Descascarar: Véase Asear ra cepa.

DESFONDAR : "cavar profundamente la tierra mezclando la de abajo con la de arriba; se hacía con arado de malacate».

DESGAJAR : (arrancar violentamente los gajos y sarmientos de la cepa).

Desgange: Véase Yema montada.

DESGRAMAR : "extirpar la grama".

DESHOJAR: "quitar las hojas que den sombra al racimo".

Desmamonar : "quitar los mamones".

DESNIETAR : "cortar los nietos".

Despalillar : "separar de la uva el escobajo cuando se procede a la pisa de aquélla; se consigue, según los técnicos, adelantar la época de clareo del mosto, mayor viveza de colorido y mejor fermentación".

Despojo : "efecto de cerner la vid, es decir, de caer la flor y quedar el frutom.

Destaluzar : "descargar la cepa de ramaje».

DesterRonar : "deshacer los terrones de tierra" \| Cf. Cuestionario Alea, 1052.

DESTETAR : “cortar los mamones» \| "cortar los mugrones por la parte que se comunican con la cepa madre» \| "cortar la cabeza del patrón injertado en yema).

DESTRIPATURRón : "jornalero que cava o ara la viña». 
DORID.1: "dícese de la ava que comienza a madurar".

ECHAR UN CIGARRO: "descansar por breve tiempo, en las viñas o en las bodegas, para tomar un bocado, echar un trago o un cigarro".

ECHIR UNA MIMM: "amarrar la yema injertada, por medio de tres anillas de palma - la última a unos 40 centímetros del suelo--, al rodrigón para evitar que el viento la desgarren.

EMRRAZIR IA CHPA: "formar la cabeza de la cepa de la cual parten los. brazos).

EMPIRRADO: "lugar cubierto de parras".

EMPARRAR: "formar un emparrado".

ENCílR: "poner rodrigones a los sarmientos para mantenerlos sobre la superficie del snelon.

ENRAIZAR: "echar raíces, agarrar".

Entritrĩo: "árbol que se planta en el centro de dos liños».

Escula: "caña que sirve para medir la distancia entre los tientos".

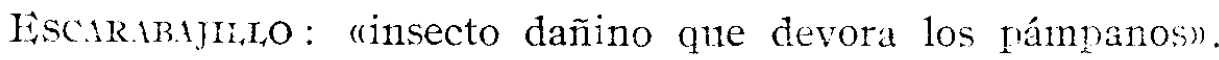

Esconajo: "parte leñosa del racimo, desprovisto ya de las uvas".

ESCOPIILI DE TOMIILA: "usada para harrer el lagar».

ESPAIIIIAR : "quitar los palillos o escobajo a las uvas".

EsIrIfA: "medida arbitraria, de caña, madera e hierro, que indica la profundidad a que hay que plantar los barbados".

Essojo: Véase Despojo.

Esourmo: "fruto que se obtiene de la vid".

Estaca: "cepa joven de dos o tres años" ! "cepa sin brazos por haberse secado, pero verde de la cabeza hacia abajo: la cepa mete entonces por donde puede) |! "soporte en la pared para colgar la capacha».

ESTADALES : "medida divisoria de la aranzada).

EsTAMBritos: Véase Estambres.

ESTANCIA : "habitación o lugar para los bueyes».

Estaquilla: "nuevo sarmiento de la cepa" " "sarmiento para plantación" || "primer sarmiento que produce, al segundo año de plantado, el sarmiento; se utiliza para barbón).

ESTAR EN IGRAZ: "dícese de la uva que no ha llegado a madurez perfecta).

Estar barbado: "dícese del sarmiento recién plantado que echa sus primeras raíces").

ESTAR BAULANDO : "abrir baúles».

ESTAR CIRNIENDO: "dejar caer la flor y conservar el fruto" \| Cf. Cuestionario ALEA, 1108. 
ESTAR COCIÉNDOSE: "dícese de la uva que, dejada en el lagar mucho tiempo sin pisarla, comienza el mosto a fermentar, aun en grano".

ESTAR CORRIDO: "(aplícase al racimo que está muy claro porque han sido mal fecundadas sus flores".

ESTAR ENCHARCADO: "dícese del terreno fangoso".

ESTAR DE ERÍA: "dícese del trozo mal labrado de las viñas).

ESTAR PINTÁNDOSE: (aplícase a la uva que empieza a colorearse».

ESTAR DE VIROTE: “dícese de la yema que va a producir racimos esie año').

ESTERCOLAR : "abonar la viña con estiércol, que se deposita en los baúles o casillas).

ESTERILla : "faja de pleita de esparto, de $12 \mathrm{~cm}$. de ancho por $25 \mathrm{~m}$. de largo, con la que se rodea y envuelve la masa de uva pisada de que se forma el pien.

ESTERRÓN : "pieza de pleita de esparto rectangular".

ESTRUJADORA : "máquina para prensar la uva, usada algunas veces en vez. del pisado").

Extender : Véase CAvabien.

FAICA: "parte del lagar que rodea la tarima, de unos dos pies de altura, dispuesta con cierta oblicuidad".

Fiel : Véase Cabeza \|| "punto, del sarmiento recién plantado, del que parten los brazos que en lo sucesivo deben formar la cabeza de la cepan.

FII OXERA : "parásito parecido al pulgón y a la cochinilla, terrible por los estragos que causa en las raíces y hojas de las cepas, reduciéndolas a polvo de color oscuron.

FogARADA : "fuertes calores y sofocos de la tierra, que suceden a una abundante lluvia).

Fogarín: "habitación con chimenea, donde los obreros se secan y descansan" \| Cf. Cuestionario AI.EA, 595.

FONDo: "tarima del lagar".

Formar LA CEPA : "dar a la cepa la forma que se desee, mediante poda u otros medios).

FRUTO : "esquilmo».

GajILlo: "ramificaciones del racimo con unas cuantas uvas".

Gajo: Véase Calita \| Cf. Cuestionario AlEA, 1118, pero en el sentido de escobajo.

Gañanía : "lugar o habitación de las personas».

Gavia : "surcos abiertos en las viñas para que en ellos escurran las aguas llovedizas de los lomos). 
Gavilla : "hacecillo de sarmientos atados entre sí, o de otra leña menuda).

GAVILLERA : "lugar donde se guardan o hacinan los sarmientos para que se sequen)!.

GOLPE LLENO : "labor cuyo objeto es impedir el endurecimiento de ia tierra; se efectúa cavando hacła adelante - a una profundidad de 18 centímetros- y allanando hacia atrás después».

Gorru Y RAJO: "labor extraordinaria -complementaria- después del golpe lleno, practicada con la azada, que se clava fuertemente en tierra para rajar o hundir la capa superficial de ésta, arrancando plantas parásitas, hierbas, raíces, etc.".

GOI,PE Y RECALO : "nuevo golpe de azada con el que se arranca la tierra que no se logró arrancar en el primer golpen.

GOTA DE MOSTO: "jugo de la uva).

GOTEAR: "dejar caer gotas".

Grama: "hierba dañina) || Pinicum Dic'ryion L.

Granuja : "uva desgranada y separada de los racimos".

GREDA : "tierra blanca y pegajosa".

GreñUeIA : "cepa joven de dos o tres años».

GUarda la VIÑA: Véase VIÑAdERo.

Guía : "tallo que sostiene Jos racimos".

HACER UN PIE: "amontonar alrededor del husillo la masa obtenida al pisar la uva; tiene forma cilíndrical.

HazA: "posesión de más de una obrada; se usa en otros cultivos de Jerez, pero no en la viña).

HFCha PASA : "dícese de la uva que, por exceso de sol, se encuentra seca, pero no lo suficiente como nara ser considerada pasan.

Hijuela : "acequia secundaria que introduce el agua en la posesión».

HOCES DE PODAR: “instrumento usado para la poda).

Hocino: "hoz de podar pequeña".

Hollejo: "pellejo de la uva, al que se debe el principio colorante del vinol).

HOMBRE DE CAMPO REDONDO: "dícese de la persona que conoce todos los cultivos por haber trabajado en ellos".

Hombre mano: "obrero de la viña que va a la cabeza de la fila y marca la norma para el trabajo de los demás).

Horouil la : “caña o palo de pino utilizado para sostener las varas de la cepa y los racimos).

HORQUILIAR : "poner horquillas a las varas de la cepa para que no se caigan con el peso de los racimos y desgajen las otras ramas". 
HORQUILLERO: "lugar donde se guardan las horquillas; generalmente en montones y lugar abierton.

HOYO PARA ESTIÉRCOL: Véase BAÚLES.

HuEso: "pepitas o semillas de la uva".

Huevecilito : "sitio de donde nacen las raíces".

HuNDIDo : "sarmiento acodado».

HUNDIR: "(acodar, enterrar un sarmiento dejando fuera su extremidad». (Era un sistema, hoy en desuso, para reponer las marras: se cogía un sarmiento largo de otra cepa, se hundía en tierra y se sacaba por la pileta donde estaba la marra. Hoy se hacen cuadrantes).

Husilio: "pieza de madera que se alza vertical en el centro del lagar, a cuyo alrededor se apila el hollejo de la uva para prensarlon.

INJERCIÓN : "acto de injertar).

INJERTAR: "operación tendente a obtener una cepa de pie americano y cabeza europea; para ello se introduce una yema o una espiga del país en un pie americano".

INJERTO DE ESPIGA : "sistema de injertar consistente en seccionar el patrón con las tijeras de podar por la parte más conveniente, después se hiende por el centro con la navaja de injertar y se le introduce la púa (que forma un ángulo de trece o quince grados) de planta del país, ligándose finalmente con rafia).

INJERTO DE YEMA : "sistema de injerto consistente en introducir una yema de planta del país en una cajuela abierta en el patrón americano».

JATO : "manta, chaqueta, blusa de tela).

JUAN DE IA VIÑA : “desgraciado que merodea por las viñas. Por extensión, desgraciado, infeliz).

Juncia : "hierba de todas las estaciones, aunque de flores estivales situadas en las axilas de las hojas" II Cyperus Longus $L$.

LABOR : "nombre genérico de las distintas tareas llevadas a efecto en las viñas)".

LABRAR: Véase CAVAR.

LADERA : "vertiente de una colina, plantada de viña" "I "explanada en la cima de un cerro, susceptible de ser plantada de viñan \| Cf. Cuestionario ALEA, 972.

LAGAR : "especie de estanque cuadrado, de madera, de tres a cinco metros de lado, cuyas paredes - falca - tienen una ligera inclinación; descansa sobre pies de madera de un metro de altura y tiene en el centro un husillo de madera para prensar los orujos; el suelo constituye un plano inclinado con objeto de que el mosto corra hacia una parte en la que existe un desagüe o piqueran). 
LAGARETa : "lagar pequeño" || "pequeña laguna que se forma en tiempos de lluvia).

LAGUNA : "dícese de un terreno inundado y fangoso".

LASTÓN : "especie de juncia que daña la cepa taladrando sus raíces" " AvENA BARBATA L.

LAVAR IA UVA: "quitar la pruina a la uva destinada al consumo de mesa, nunca a la reservada para la elaboración de vinosn.

LEÑA : "madera" || "parte del sarmiento por donde no circula la savia".

I EEVANTAR LAS VARAS : "alzar las varas que han de dar fruto, por medio de horquillas, para evitar que al contacto con la tierra húmeda se pudra la uval.

LeVante: "viento del Este» \# Cf. Cuestionario AlEA, 911.

LIÑAR: "alinear las vides al plantarlas, de modo que forman liños).

LrÑo: "hilera de cepas, plantadas una detrás de otra a una distancia media de un metro y medion.

LIÑO MADRE : "cepa que cierra la viña».

LLORAR: "perder savia la cepa por los cortes hechos en la poda".

LiLORO: "derrame de savia por los cortes de los brazos podados".

MACHEA: "dícese de la cepa que da mayor cantidad de agracejos que de uvas perfectas").

MADURA : "uva que ha alcanzado su total desarrollo, pudiendo, por tanto, ser vendimiadan.

Majuelo: "cepa joven de dos o tres años" "| "pago recién plantado de viñas" || Cf. Cuestionario ArteA, 1097.

MAI, DESPOJO: "fecundación, numéricamente deficiente, de la flor».

MAMONES : "hijuelos o retoños que nacen de las raíces de las cepas» || "sarmientos, que hay que cortar, de la cepa americanan.

MANGIA : "enfermedad de las cepas producida por un insecto que, con sus picaduras, ocasiona la pérdida de savia».

Mantua PILA : "variedad de uvas: grandes, muy redondas, algo doradas y muy tardías".

MARCADO : "señalamiento que se hace en el terreno para proceder a la plantación de las cepas; se lleva a cabo con una cadena, con motas a la distancia a que han de plantarse las cepas, clavando los tientos donde la mota indique. Se emplean dos sistemas de marcado: Marco Real y Tres Bolillosm.

MARCAR : "Operación que sigue a la de allanar, y consiste en hacer el marcado del terreno). 
MARCo REAL : "sistema de plantación en el que cada cepa ocupa el vértice de un cuadrado; los liños forman, pues, líneas paralelas perpendiculares).

MARRA : "cepa o plantón perdido".

MARRANA : "tuerca de madera, que se desliza a lo largo del husillo y que descansa sobre dos aros provistos cada uno de un mango o palanca ajroximadamente de un metro").

MarRanos : Véase Queso.

Más enVEnADO: "dícese del sarmiento que disfruta de más savia".

MAYETO: "propietario o arrendador de una pequeña viña".

METEDORES DE UVA: "los que llevan la uva desde la viña o desde el almijar hasta la bodegan.

METER : «brotar».

METERSE EN AGRAZ : (tomar la uva ácidos para empezar a madurar).

Metidas : “brotes de la viña) || Cf. Cuestionario Ar.EA, 1049.

MOLEDOR: Véase Esstrujadora.

MOSCATEL: "variedad de uvas: grandes, blancas, de sabor almizcleño».

Mота : "señal que se pone a la cuerda o cadena empleada en el marcado de la viña y que indica el sitio en que hay que clavar el tiento".

MUGRONEAR: "clavar en tierra las puntas de los sarmientos para que echen raíces) \|| ("hundir).

MUGRón DE CABESTRILLO: "mugrón que, por no alcanzar hasta el cuadrante de la marra, se entierra dejándole la punta fuera, punta que comúnmente alarga en el año siguiente para alcanzar la marran.

MUGRONES : "sarmientos que, sin separarlos de la cepa, se introducen en la tierra $y$ en ella enraizan, llegando a formar una nueva planta una vez que se corten y separen de la madren.

MUÑECA : "casta de uva".

NAVAJA DE INJERTAR: "navaja generalmente barbera utilizada para esta faena).

NiEBr,A : (nube pegada al suelo".

Nreto: "ramitos que salen de los sarmientos principales).

NIÑo: Véase ZAGAL.

OBRERO: “jornalero eventual en una viña, que trabaja a las órdenes inmediatas del capataz).

ORUGA : "insecto que ataca a las viñas).

Orujo: "hollejo de la uva pisada" "| Cf. Cuestionario AleAa, 1137.

PAGo: "extensión de terreno plantado de viñas». 
PAIA : "instrumento de madera utilizado para formar el pie con la ayuda de unas escobillas de tomillo; úsase también dentro del lagar para amontonar o esparcir la ivan.

PaLII, : : "racimo al que se le quitan las uvas" (es más frecuente que escobajo).

PALM.1: "planta de la que se hace la pleita para redores, capachos, ctc.». Priomio: "variedad de uvas: medianas, muy sabrosas y azucaradas; racimos grandes, dorados o verdosos, según les dé el sol o la sombra".

PÁmpanas: "metidas de la cepa».

Pámpinos: "hojas de la vid" |f "sarmiento verde, tierno y delgado, o pimpollo del extremo del sarmiento".

PAPAFRI'A : "hombre infeliz y despreciable».

PARRA : "vid aérea levantada artificialmente".

PARRAL: "plantación de parras» "terreno plantado de parras".

PARRón : "parra silvestre» || "uva silvestre».

PASA : "uva secada al sol».

PATIILA: "nacimiento de las raíces, huevecillo".

PATrón : "planta americana en la cual se injerta la pianta del país; es, pues, el portainjerto» || "barbón ya más hecho» || "dueño de la viña arrendada).

PEDRo JIMÉnez : "variedad de uvas: doradas, traslucientes, blandas, sumamente dulces).

Peirlizcar la UVA: "picat en el racimo".

PERRUNO DE ARCO: "variedad de uvas: gruesas, duras, traslucientes, color amarillento; racimos abundantes».

PETO: "parte ancha y pesada de la hoz de podar, que sirve para podar sacudiendo, como si fuera de golpe, los brazos secosm.

Picón : Véase Cerrnidura.

PIE DE YEMA : "mosto procedente del primer pien.

PIIETA : "poza u hoyo cuadrado o redondo hecho para detener las aguas al pie de la cepa).

PINchauva : "hombre infeliz y despreciable).

PINTAR LA CEPA : “lavar las cepas - con brocha o muñequilla de trapo empapada en sulfato de hierro- para destruir las esporas) (también se pintan los cortes de la cepa después de la poda).

PIN'TONA : "dorada»).

PiQUERA: "parte del lagar por donde sale el mosto. Va provista de un colador de alambre o canasto, para impedir que el mosto arrastre el hollejo de la uval. 
Pisadores: "obreros que trabajan en el lagar durante la vendimia, en mangas de camisa, con calzones cortos ceñidos hasta la mitad de los muslos y zapatos de pisa groseramente claveteados por las suelas. Se colocan en filas, agarrados unos a otros, recorriendo el lagar en un continuo y cadenciado movimiento de vaivén. Suelen emplearse cuatro pisadores por lagar, y se apoyan en el mango de una pala de madera que sirve para remover de vez en cuando la uvan.

PISAR: "estrujar la uva los pisadores con los pies".

Pisones: "mazos de mano, de madera, con que se apisona la uva Pedro Ximénez).

Plantar a LA BARRa: "sistema de plantación de barbados que se lleva a efecto con una barra; el barbado ha de quedar metido en tierra hasta el punto de arranque de su brotación, y, por ello, se requiere que la tierra esté bien desfondada).

Plantrjo : "planta, barbón".

Plantón : "cepa nueva".

PleitTa : "tira de tejido de esparto o palma".

POCEjón: "hoyos hechos en el suelo para que viertan en ellos el mosto de los lagares».

PODA : "acto de podar».

PODA EN BERza: Véase CASTRA.

PODA CORTA : "cuando a los sarmientos se les deja una, dos o tres yemas". PODA LARGA : "cuando a los sarmientos se les deja más de tres yemas».

PODADORES: "obreros que podan la viña).

PODAR: (cortar los sarmientos» \| Cf. Cuestionario AI.EA, 1103.

PONER viñas a CAJONES : "abrir de dos en dos los hoyos para las cepas, en vez de uno en uno».

PonIENTE: "viento procedente de Sanlúcar de Barrameda".

POŚTURA : "forma o sistema de plantar las cepas").

PRENSAR : "apretar la uva pisada en el husillo, haciendo un pie con ella» \| "someter el orujo —en capachos especiales- a la presión hidráulica".

PRIMER PIE : “masa de uva pisada que por primera vez va a ser sometida a la operación de tirarn.

PRIVAR EL PLANTIJO : "agarrar, enraizar, arraigar la planta".

Pruina: "polvillo de materia aérea que cubre la uva y que actúa como agente catalizador indispensable en la fermentación del vino; por ello hay que suspender la corta de la uva si llueven.

PÚA: "parte que se introduce en la cepa americana, en el injerto de espiga)).

Pulgares : “brazos de la cepa». 
Pulgón: "insecto nocivo que se adhiere a los brotes y al escobajo" \| ArTiCa Ampelophaga y Aphis Vitrs.

QUEMADERO: "lugar donde se queman los sarmientos».

QUEMADO: "mancha de la uva ocasionada por la acción directa del sol en días de calma).

QUeso: “dos piezas de madera machihembradas que se colocan encima del pie, en el husillon.

QUITAR RODRIGONES : "quitar los rodrigones a los sarmientos después de la vendimia).

RABILIO : "pedicelo o pequeño cabo que une la uva al racimo".

RACimo: "conjunto de uvas unidas por los rabillos a un tallo que pende del sarmienton.

RACimo APIÑADO: "racimo cuyas uvas están muy juntas).

RAEDERA : “instrumento para limpiar las herramientas».

REBUSCADOR: "campesino que se dedica, en su provecho, a recoger el rebuscon).

REAUSCAR : “recoger los rebuscos que quedan en la cepa después de vendimiada una viña).

REBuscos: "racimos que nacen de los nietos)" $\mid$ "fruto que queda en las viñas después de terminada la vendimia" || "acto de rebuscar).

REBUSQUILLOS: "(uvas que aún quedan en la viña después del rebusco». ReCalar los Gol,Pes : Véase Golpe y ReCalo.

RECASTRA : "acto de recastrar»).

RECAS'TRAR : "Volver a castrar; entre castra y recastra hay un mes o cuarenta días de diferencia).

RECOGER CAÑAS: Véase RECOGER LOS RODRIGONES.

RECOGER PALILLOS: ("retirar los restos que queden de la sarmienta).

RECOGER LOS RODRIGONES : "retirar los rodrigones, inútiles ya por haber cumplido su misión de sostén; se hace esta faena en octubre».

RECOGER IA SARMIENTA: Véase SARMENTAR.

RECOGIDA DE HORQUIILAS: "acto de retirar las horquillas).

REDOR : "esterilla redonda, tejida de esparto, con cuatro asas, y cuya función es idéntica a la del capacho».

REHORQUILLAR: "poner de nuevo las horquillas que se han caído".

Rentero: Véase Colono.

REPARTIR RODRIGONES: Véase ENCAÑNA.

REPASAR : “revisar los plantones para reponer los que se hayan yerdido; es faena que se hace en enero" |l "volver a pisar la uva antes de prensarla; no siempre se hace). 
REPASAR REPOSICIÓN : "revisar los mugrones que se habían plantado en las marras).

REPASO DE LAS YEMAS : "faena consistente en comprobar el estado en que se encuentran los injertos de yemia: si continúan ligados o fajados con la rafian.

REPOSICIón : “sustitución de las cepas que no dan frutos por mugrones, arrancando aquéllas y abriendo un cuadrante para éstos".

RETENIDA: "paredes de piedra que se hacen en el campo para contener el terrenom.

RIPARIA : "planta del país» \|C Cf. Cuestionario ALEA, 1907, pero con el sentido de "viña joven".

ROcraR : "desparramar la tierra con la azada de manera que quede llana la superficie del terrenon.

RODRIGón : “caña o estaca que se clava al pie del sarmiento y a la cual éste se amarra con cbjeto de mantenerlo derecho hasta que robustecido pueda ya sostenerse en la posición necesaria para su cultivon || Cf. Cuestionario ALEA, 1495.

ROLEAR : "granar poco los racimos, dar uva menuda).

RoLón : (flojo, holgazán».

Rondino: "viento entre Norte y Este» \| Cf. Cuestionario ALEA, 911.

Rosquilla : "insecto negro, cubierto de vello gris, con los élitros y partes de las patas de color rojizo, teniendo finamente punteada su cabeza y tórax; roe las hojas, sin que las lesiones que causa tengan gran importancia; peligrosas sus larvas, que se fijan en las raíces y hacen surcos longitudinales) \| ADoxus vitis.

RozAR: "limpiar de yerbas y grama el terreno de las viñas con un hocinon.

Salitroso: "dícese del terreno estéril).

SARMENTADOR: (el que recoge los sarmientos podados).

SARMENTAR: "recoger los sarmientos cortados".

SARMIENTA : "recogida de los sarmientos cortados" \| "conjunto de sarmientos cortados).

SARMiento: (cástagos o ramas de la cepa, largos, delgados y nudosos, en los que salen los racimos).

SARMIEnTo ACODADo: "sarmiento al que se le entierra el tallo doblado dejando fuera la extremidad; no se separa de la cepan.

SAvia : "Sustancia acuosa vital que corre por el corazón de la planta".

SECO : "superficie de la cepa que va por detrás del verde".

SEGUNDO PIE : “masa de uva pisada que por segunda vez va a ser sometida a la operación de tirar). 
Señorito: Véase Amo.

Sesenta y uno: "vidueño de color rubio".

Sierpes: Véase Mamones.

SIETE COLORES : "tierra estéril color ceniza, barro claro, añil, plomo, etc.». SOLEAR: "exponer los racimos ya cortados extendidos en capachos o redores durante 24 horas o varios días - según los vinos que se deseen obtener - a la acción del sol; de noche se guardan los racimos bajo techado o se protegen con redores superpuestos o quedan expuestos en el almijarn.

SOLEO : "acción de solear".

Sombrajo: "bienteveo que no está levantado sobre los altos palos, sino que se encuentra sobre el suelon.

SUERTE: "viña separada de otras por las lindes" \| Cf. Cuestionario Alea, 1018.

Sulfatar: Véase Pintar la CEPA.

SULFATO DE HIERRO: "producto químico con el que se pintan las cepas después de la poda".

SUR BAJO: "viento del SE.").

TAJO: "corte que se da con la azada o con algún otro instrumento".

TAjón: “tierra caliza (80 por 100), que no se deshace ni esponja, sino que cada vez adquiere mayor dureza; se utiliza para cal; suele aparecer al abrir hoyos para plantar las vides, a un metro de profundidad).

Temprinilia: Véase Palomino.

TENER I.EÑA: "dícese de la cepa muy frondosa".

TERRENO: "tierra a propósito para plantar cepas».

TIENTO: "caña o vara que sirve de orientación para fijar los sitios donde se han de plantar los sarmientos sin desviarse de las distancias correspondientes ni perder la simetrían.

TIERRA I,USTRILLOSA : "tierra de color negruzco, con guijas".

Tijeras: "herramienta utilizada para la poda".

TIJERETA: "racimo de uva que no se ha desarrollado, ni ramificado, ni cubierto de flores, quedando como cordelillo que se retuercen.

TINETA: “recipiente de madera, en forma de artesones, en el que se coloca la uva al cortarla de la cepa y que se deja en el almijar para que se solee; tiene una capacidad de 12 a 14 kilogramos).

TIRADERAS: "amarrija con la que los pisadores se atan la mano derecha a la palanca del husillo para hacer el pies.

Tiradores: "los obreros que hacen el pie y luego tiran". 
TIRAR : "apretar el pie en el husillo empujando la palanca con las manos, una de las cuales - la derecha- está atada a ella con las tiraderas". Tomiza : "cuerda o soguilla de palma o esparto".

TongA : "cada una de las dos mitades en que -para ser pisada de una vez- se divide la carretada).

TONTAja: Véase JUAN DE LA VIÑA.

ToscA : "tierra de albariza, cuya proporción de caliza nunca es inferior al 40 por 100 ; se deshace, esponja y divide fácilmente, siendo la tierra mejor para el cultivo de viñas».

Travesaños: "cuatro o cinco palos que se colocan encima del queso".

TRES BOLILLOS: "sistema de plantación en el que con cada tres cepas se forma un triángulo equilátero; los liños, pues, forman líneas paralelas y diagonales).

TRIÁNGULO: "labor consistente en abrir, entre cepa y cepa, una pileta o casilla honda donde se entierra el estiércol los años que la viña se estercola).

Uva : "fruto de la vid".

UVA EN AGRAZ: "uva verde".

UVA APLUMADA : "la que no llega a madurar".

UVA CARRASQUEÑa : "uva desmedrada, degenerada de casta".

UVA DE CUELGA : "la destinada a consumirse en invierno y que se conserva colgada y bien aireada).

UVA MOLLAR: Véase CaÑocazo.

UVA PILA: Véase MANTUA PILA.

UVA DE VERDEO: "uva para comer».

VALIENTE: "dícese de la cepa esquilmeña).

VARA : "sarmiento que lleva fruto".

VARA NUEVA : "sarmiento de seis a siete yemas".

VARA DE UVA: "sarmiento que lleva uva".

VARIEDAD DE UVA: "especies o castas".

VÁsTAGo: ("rama fuerte de la cepa).

VENCEJO DE PALMA : "dos puntas de palma entrelazadas".

VENDIMIA : "recogida de la uva) || "conjunto de los frutos recolectados» || "fecha en que se hacen las operaciones necesarias para cortar la uva, transportarla a los lagares y pisarla; duran aproximadamente un mes: septiembre).

VENDIMIADORES : "hombres o mujeres que se dedican y ocupan de las faenas de la vendimian.

VENDIMIAR : ((recoger el fruto de las viñas). 
VENITA DE LA UVA: "porción de carne o pulpa que queda adherida al palillo cuando éste es arrancado de la uval.

VERDEAR : "vender la uva para consumirla fresca).

VERDES : "parte de la cepa donde se encuentran las yemas útiles para poblar y formar la cabeza).

VERDE SECO : "brazo seco de la cepa).

VERDIONA: "aplícase a la uva que es o está verde».

VIDUEÑO : "especie, casta de las vides" || "grupo de cepas que producen la misma calidad de vino's.

VIDUEÑ̃O LOCO: "vidueño cuyos racimos son escasos o nulos).

VIENTO NORTE: "viento procedente de Sevilla).

VIENTOS DE ABAJO: "vientos del mes de abril».

VIENTO DEL SUR: "viento procedente de Cádiz".

VILLARES : "tierras de buhedo en que aparecen restos de antiguas construcciones).

VINAGRILLO: "descanso de media hora que se concede a los obreros durante el trabajo y en el que se toma la catana, puesta por el amo, excepto el pan».

VIÑA : "campo de vides" \|Cf. Cuestionario ALEA, 1096.

VIÑA DE AFUERA : "viñas colocadas en tierras de albariza que por estar situadas en las afueras -las albarizas más próximas están a 5 kilómetros de Jerez- se designan con este nombren.

VIÑAS DE BARRO: "viñas plantadas en tierras de barro".

VIÑA ENGUACHIRNADA: "viña encenagada".

VIÑA DEI, PAÍS: "planta del país, es decir, planta europea".

VIÑADERO: "hombre que, desde el bienteveo, vigila la viña para evitar desmanes de desaprensivos).

VIÑEDO : "plantío de viñas".

VIÑISTA : "trabajador en las viñas).

VIVERO: "lugar donde se siembran los sarmientos que luego se utilizan en las plantaciones).

VIZCONDE: "obrero que sigue al hombre mano".

YEMA : "brotes de la vid» \| "botón abierto".

YeMA CASQUERA: "la que está en el nacimiento de! sarmiento".

YEMA LLANA: "la que sale horizontal al suelo".

YEMA MONTADA : "la que tira al seco".

YEMA DE VERDE: "brazos de la cepa vieja».

YEMA DE VIROTE: Véase ESTAR DE VIROTE.

YEMERO: "cada trozo del sarmiento que tiene una yema". 
ZAGAL : "muchacho destinado en las viñas a los menesteres más humildes) $\|$ Cf. Cuestionario ALEA, 164.

ZANJA : "hoyo o gavia larga, que se abre en las viñas enclavadas en colnnas o cañadas para facilitar los desagües en épocas de lluvian.

ZAPATOS DE PISA : "calzado de cuero de vaca, con clavos combinados en la suela, de pala más alta que el corriente - casi una media bota-, usado por los pisadores".

ZARANDA : "cedazo con que se criba el pie -después de obtener la yemaya deshecho para quitar el palillo" || "cesta de mimbre que se pone en la piquera del lagar para colar el moston.

ZARROSTRADA: "mal cuidado de la viña, que luego sufre las consecuencias").

ANTONIO ROLdDÁN 